\title{
Wavefront propagation in a competition equation with a new motility term modelling contact inhibition between cell populations
}

\author{
By Jonathan A. SherRatT \\ Centre for Theoretical Modelling in Medicine, Department of Mathematics, \\ Heriot-Watt University, Edinburgh EH14 4AS, UK (jas@ma.hw.ac.uk)
}

Received 3 November 1999; accepted 16 March 2000

Linear diffusion is an established model for spatial spread in biological systems, including movement of cell populations. However, for interacting, closely packed cell populations, simple diffusion is inappropriate, because different cell populations will not move through one another: rather, a cell will stop moving when it encounters another cell. In this paper, I introduce a nonlinear diffusion term that reflects this phenomenon, known as contact inhibition of migration. I study this term in the context of two competing cell populations, one of which has a proliferative advantage over the other; this is motivated by the very early stages of solid tumour growth. I focus in particular on travelling-wave solutions, corresponding to moving interfaces between the two cell populations. Numerical simulations indicate that there are wavefront solutions for wave speeds above a critical minimum value, and I present linear analysis that explains the selection of wave speeds by initial conditions. I obtain an approximation to the shape of these waves for high speeds, and show that the minimum speed arises via quite new behaviour in the travelling-wave equations, with the proportion of cells of each type approaching a step function as the wave speed decreases towards the minimum. Exploiting this structure, I use singular perturbation theory to investigate the wave shape for speeds close to the minimum.

Keywords: travelling wave; competition; reaction-diffusion; contact inhibition

\section{Introduction}

Diffusion has a long history as a model for spatial spread in biological systems. In ecology it was first used by Skellam (1951), and has subsequently become widespread in work on both invasion and patterning (reviewed in Okubo (1980) and Murray (1989)). For motile cell populations, diffusion has been used very successfully for ca. 30 years (see, for example, Keller \& Segel 1971), with applications including capillary network growth (Chaplain \& Stuart 1993), developmental pattern formation (Murray \& Oster 1984), and wound healing (Sherratt \& Murray 1990). In a number of more recent models, diffusion terms have been included for each of two or more cell populations (Sherratt \& Nowak 1992; Pettet et al. 1996; Gaffney et al. 1999). This is entirely appropriate for cell populations in which the cells are widely separated; a natural example is fibroblasts in the dermal layer of the skin, which are typically separated by about ten cell diameters. However, diffusion is qualitatively inaccurate 
for close-packed cell populations such as epithelia, where one cell is in direct contact with its neighbour.

For close-packed cells, a reaction-diffusion equation proves to be a good model for a single population (Sherratt \& Murray 1990; Chaplain \& Stuart 1991; Höfer et al. 1995), but for interacting populations, diffusion terms would imply that the populations are able to mix completely, with the movement of one cell type unaffected by the presence of cells of the other type. The reality is exactly the opposite: cell movement is typically halted by contact with another cell. This phenomenon is known as 'contact inhibition of migration', and is very well documented in many types of cells (see, for example, Abercrombie 1970; Huttenlocher et al. 1998); however, to my knowledge, no mathematical model incorporating contact inhibition has previously been described. In this paper, I propose such a model via a simple extension of linear diffusion. I illustrate the model by considering the case of two competing cell populations, a problem motivated by the very early stages of tumour formation. In particular I study travelling-wave solutions of the model, demonstrating unusual wavefront behaviour.

Interacting cell populations arise in many biological situations; I take the early growth of solid tumours as a simple example. The development of a fully malignant tumour is a complex, multi-stage process with many possible developmental sequences. However, in the vast majority of cases the initiating step is a mutation, causing one cell in a tissue to divide more rapidly than its peers. This rapid cell division gives rise to a small benign tumour that usually poses no health problems in itself, but may progress towards malignancy as a result of further mutations. Benign tumour growth itself occurs through well-known stages, with the development of a 'necrotic' core of dead cells within a proliferating cellular rim, possibly surrounded by a 'capsule' of dense protein fibres; these processes have been modelled by a number of authors (see, for example, Ward \& King 1997; Byrne \& Chaplain 1997; Perumpanani et al. 1997). Preceding these stages is the simple initial competition process between tumour cells and surrounding normal tissue cells. This has been modelled by a number of authors using both ordinary differential equation (ODE) (Wheldon 1975; Michelson \& Leith 1991; Gatenby 1995, 1996) and partial differential equation (PDE) (Sherratt 1993; Gatenby \& Gawlinski 1996) models, showing how competition parameters affect initial tumour development. However, a detailed study of this process requires a spatial movement term that reflects contact inhibition between cells from the two populations. This is because the vast majority of cancers are carcinomas, arising in the surface layers of tissues (epithelia), where cells are closely packed.

The basis of a diffusion model for the spatial spread of a cell population is that cells tend to move in a direction in which they have a free edge, so that the overall cell flux can reasonably be taken to be proportional to the negative gradient of cell density. There is no quantitative basis for this movement term, but it has been applied to a single-cell population with great success in a variety of contexts. For competing cell populations, with densities $u(\underline{r}, t)$ and $v(\underline{r}, t)$ say, the overall cell flux (of both populations) can similarly be taken as $-\nabla(u+v)$. Of this flux, a fraction $u /(u+v)$ will be cells from the $u$ population, so that the flux of cells from this population is simply $-[u /(u+v)] \nabla(u+v)$, with the flux of cells from the $v$ population given similarly. These are simple expressions, but, crucially, they reflect the fact that the movement of one population is inhibited by the presence of the other. Note that the 
difference between this new flux term and that for linear diffusion is

$$
-\left[\frac{u}{u+v}\right] \nabla(u+v)+\nabla u=\frac{-u^{2}}{u+v} \nabla\left(\frac{v}{u}\right) .
$$

Thus, the two terms are equivalent when the ratio $v / u$ is constant, and also the difference tends to zero as $u$ and $v$ become small. Both of these properties confirm intuitive expectations.

I will consider these new terms for cell flux in the context of the following simple model for initial tumour growth:

$$
\begin{aligned}
& \frac{\partial u}{\partial t}=\frac{\partial}{\partial x}\left[\frac{u}{u+v} \frac{\partial}{\partial x}(u+v)\right]+u(1-u-v), \\
& \frac{\partial v}{\partial t}=\frac{\partial}{\partial x}\left[\frac{v}{u+v} \frac{\partial}{\partial x}(u+v)\right]+v(\gamma-u-v) .
\end{aligned}
$$

Here $u$ and $v$ denote densities of normal and tumour cell populations respectively, which have been rescaled so that $u \equiv 1$ in normal tissue. The term $-(u+v)$ in the kinetics represents the decrease in the cell division rate due to crowding, and the constant $\gamma$ (greater than 1) represents the proliferative advantage of the tumour cell population. Here and throughout the paper I restrict attention to a one-dimensional spatial domain. The kinetics of (1.1) are exactly of the 'LotkaVolterra' competition type (Lotka 1925; Volterra 1926), which have been very well studied in ecological applications (see Murray (1989) for a review). They are certainly not a quantitatively accurate representation of tumour cell kinetics, and the importance of (1.1) is as a generic model of the underlying competition process, providing a simple context in which to investigate the implications of my new movement term.

The remainder of this paper is organized in the following manner. In $\S 2$, I discuss numerical simulation of (1.1) and present linear analysis suggesting how the wave speed depends on initial data. In $\S 3$, I consider the shape of rapidly moving waves. In $\S 4$, I show that there is a minimum possible speed, and, finally, in $\S 5$ I discuss the shape of waves with speeds close to this minimum.

\section{Wavefront solutions}

A simple illustration of contact inhibition effects in (1.1) is provided by the evolution of two initially separated populations of the two cell types in the limiting case $\gamma=$ 1 ; in this case the two cell populations have identical properties. Mathematically, we formulate this problem by considering an infinite spatial domain with initial conditions

$$
u=\frac{1}{1+\exp \left\{-\xi_{u}\left(x-X_{u}\right)\right\}}, \quad v=\frac{\gamma}{1+\exp \left\{+\xi_{v}\left(x-X_{v}\right)\right\}}
$$

$\left(\xi_{u}, \xi_{v}>0\right)$; provided $X_{u}$ is significantly greater than $X_{v}$, this gives two separated populations. Note that step function initial conditions, which are commonly used in cell biology models, are not permitted here, since the equations (1.1) are undefined when $u=v=0$ : thus, $\xi_{u}$ and $\xi_{v}$ can be arbitrarily large, but the limiting case of both being infinite is not permitted. This restriction is not a significant one since 
the use of a diffusion term for cell movement implies that the density can only be zero instantaneously away from a boundary; however, care is required in numerical solutions when $u$ and $v$ are both small.

A simulation of (1.1) with initial conditions (2.1) and with $\gamma=1$ is illustrated in figure $1 a$. The two initially separated populations move towards one another, with wavefronts of $u$ and $v$ moving in the negative and positive $x$-directions, respectively. However, as the fronts approach one another, contact inhibition takes effect and the movement slows, until the fronts finally come to a halt at a steady state in which $u+v \equiv 1$, with the two cell populations immediately adjacent but only mixed at their peripheries. This is exactly what happens when actual cell populations move together, and is quite different from solution of the corresponding equations with linear diffusion as the motility term for $u$ and $v$, in which case the two populations would continue to mix, reaching equilibrium only when $u \equiv v \equiv \frac{1}{2}$. Note that the steady-state profile with $u+v \equiv 1$ depends on the details of the previous spatiotemporal evolution, since any solution with $u+v \equiv 1$ is an equilibrium (when $\gamma=1$ ). Calculation of this profile is, thus, a challenging problem that I have not attempted.

When $\gamma>1$, the initial behaviour is very similar to the $\gamma=1$ case, with fronts of $u$ and $v$ moving towards one another. However, as they come together, the $v$ wave continues to advance, but more slowly, while the $u$ wave changes direction and moves in parallel with that of $v$ (figure $1 b$ ). This corresponds to an invasion of the $u$ population by $v$, and is exactly the behaviour seen in the very early stages of a carcinoma: the tumour cells, which are dividing more rapidly as a result of a mutation, develop as a dense ball of cells that replaces surrounding tissue.

The remainder of this paper is concerned with the speed and shape of these wavefronts in which the $v$ population advances with the $u$ population receding in parallel. Numerical experimentation indicates that, with $\gamma$ fixed (greater than 1), there is a family of such solutions, with the wave speed depending on $\xi_{v}$ (but independent of $\left.\xi_{u}\right)$. Such a dependence of wave speed on the decay rate of initial data has been known for many years in scalar reaction-diffusion equations such as the Fisher equation (Rothe 1978), and has been demonstrated in a number of reaction-diffusion systems (Freidlin 1983; Dale et al. 1997; Marchant et al. 2000). To study it in this case, I look for solutions that are functions of the travelling-wave variable $z=x-c t$, with $u(x, t)=U(z)$ and $v(x, t)=V(z)$. It is most convenient to formulate the equations in terms of $V(z)$ and $N(z) \equiv U(z)+V(z)-1$, which gives

$$
\begin{aligned}
N^{\prime \prime}+c N^{\prime}-N(1+N)+(\gamma-1) V & =0, \\
{\left[\left(\frac{V}{1+N}\right) N^{\prime}\right]^{\prime}+c V^{\prime}+V(\gamma-1-N) } & =0,
\end{aligned}
$$

where a prime denotes $\mathrm{d} / \mathrm{d} z$. For the wavefronts I am considering, $U \rightarrow 0$ and $V \rightarrow \gamma$ behind the wave, while ahead of the wave, $V \rightarrow 0$ and $U \rightarrow 1$, so that $N \rightarrow 0$. Linearizing about this latter equilibrium gives

$$
\begin{aligned}
N^{\prime \prime}+c N^{\prime}-N+(\gamma-1) V & =0, \\
c V^{\prime}+(\gamma-1) V & =0 .
\end{aligned}
$$



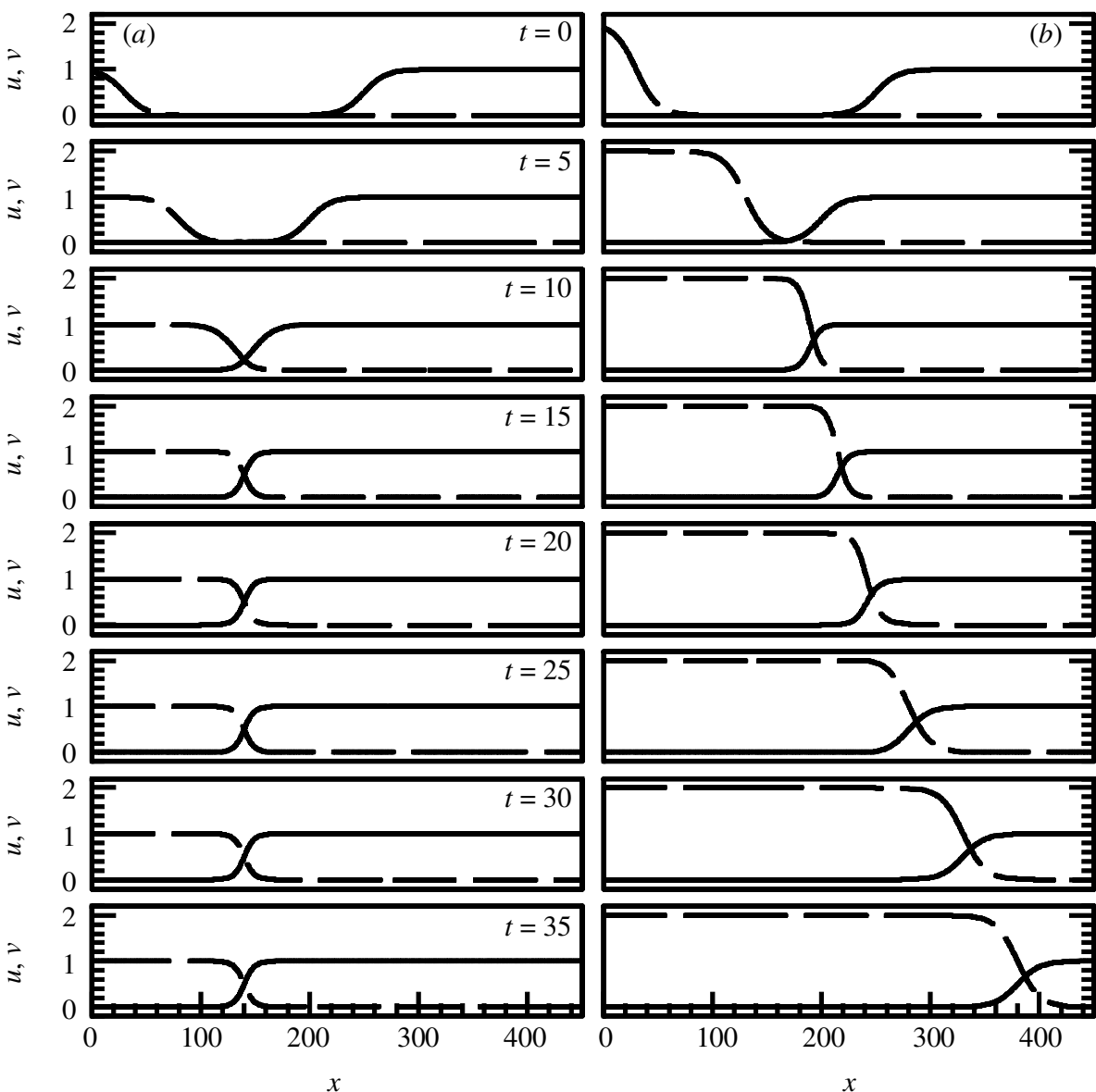

Figure 1. Typical solutions of the model equations (1.1) subject to (2.1). The two cell populations $u(-)$ and $v(---)$ are initially separated, and move towards one another. The movement slows as the populations come together, because of contact inhibition, and when $\gamma=1(a)$, the wavefronts become stationary, with $u+v \equiv 1$. However, when $\gamma>1$, the wavefront for $v$ continues to advance, in parallel with a receding wave of $u$. This corresponds to the movement of adjacent cell populations, and a study of this movement is the main focus of the paper. The parameter values are $\xi_{u}=\xi_{v}=0.1, X_{u}=250, X_{v}=30$ and $(a) \gamma=1,(b) \gamma=2$. The times indicated apply to both $(a)$ and $(b)$. The equations were solved numerically by discretizing in space to give a system of coupled ODEs, which were solved using a standard stiff differential equation solver (Brown et al. 1989); I found that the most efficient scheme is given by writing the movement term as $\left[\left(u_{x x}+v_{x x}\right) u /(u+v)\right]+\left[\left(u_{x}+v_{x}\right)\{u /(u+v)\}_{x}\right]$ and calculating derivatives using central difference approximations.

Thus, to leading order ahead of the wave,

$$
\begin{aligned}
& V=V_{0} \exp [-z \cdot(\gamma-1) / c] \\
& N=\frac{c^{2}(\gamma-1)}{c^{2} \gamma-(\gamma-1)^{2}} \exp [-z \cdot(\gamma-1) / c]+N_{0} \exp \left[-z\left\{c+\sqrt{c^{2}+4}\right\} / 2\right],
\end{aligned}
$$

where $V_{0}$ and $N_{0}$ are constants. 


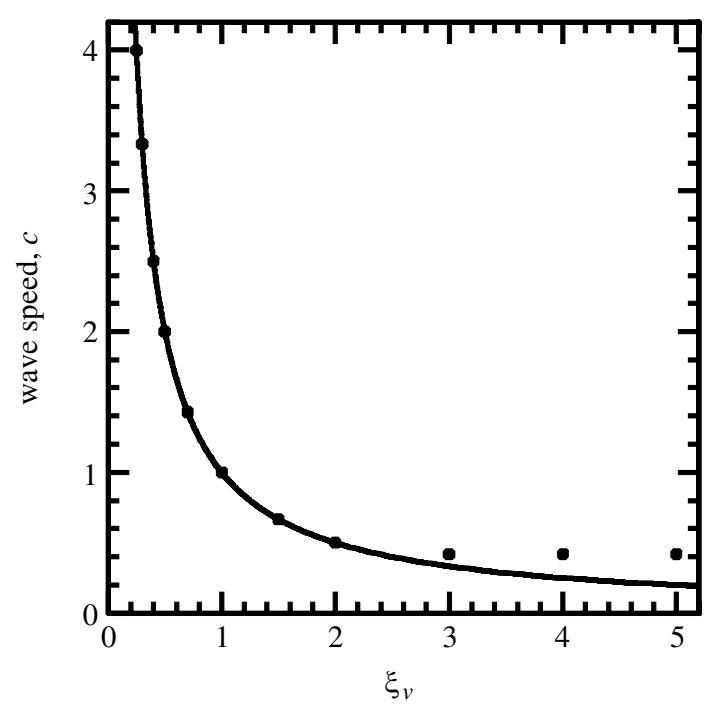

Figure 2. An illustration of the variation of wave speed $c$ with initial decay rate $\xi_{v}$ in solutions of (1.1) subject to (2.1). The points show speeds calculated from numerical solutions of the PDEs, as discussed in the legend to figure 1 , and the solid line is a plot of $c=(\gamma-1) / \xi_{v}$, as predicted by linear analysis. The comparison is extremely good except for high values of $\xi_{v}$; the behaviour for such values is discussed in $\S \S 4$ and 5 . The case illustrated is for $\gamma=2$.

Based on experience with other wave selection problems, one anticipates that the wave speed will be determined by the condition that the decay rate ahead of the $v$ wave will be the same as that of the initial data, that is

$$
c=(\gamma-1) / \xi_{v} .
$$

This is confirmed by numerical solutions for a wide range of values of $\gamma$ and $\xi_{v}$ (figure 2), except for particularly high values of $\xi_{v}$, which will be discussed later.

The form of $N$ ahead of the wave needs more careful consideration, however. The first term in $(2.4 b)$ dominates the second for large positive $z$ provided

$$
(\gamma-1) / c>\frac{1}{2}\left[c+\sqrt{c^{2}+4}\right],
$$

and, when $\gamma>1$, this simplifies to $c>(\gamma-1) / \sqrt{ } \gamma$. Using (2.5), this corresponds to $\xi_{v}<\sqrt{ } \gamma$. Thus, waves moving faster than $(\gamma-1) / \sqrt{ } \gamma$ have $V$ and $N$ decaying in parallel ahead of the wave, so that $U$ approaches 1 , from below, at the same rate as $V$ approaches 0 ; this is again confirmed in numerical solutions. However, for slower waves, $V$ decays more rapidly than $N$. Straightforward calculation of the eigenvectors of (2.3) at $N=V=0$ shows that $N$ approaches zero from above. Since $V \ll N=U+V-1$, it follows that the wave shape has a qualitative change as the speed decreases through $(\gamma-1) / \sqrt{ } \gamma$, with $U$ tending to 1 from above ahead of the slower waves. The form of wavefronts for three different speeds is illustrated by the numerical simulations in figure 3 .

\section{Wave shape for high wave speeds}

For waves moving at high speeds, the approximate form of the wave shape can be determined by adopting an approach developed by Canosa (1973) for the Fisher 


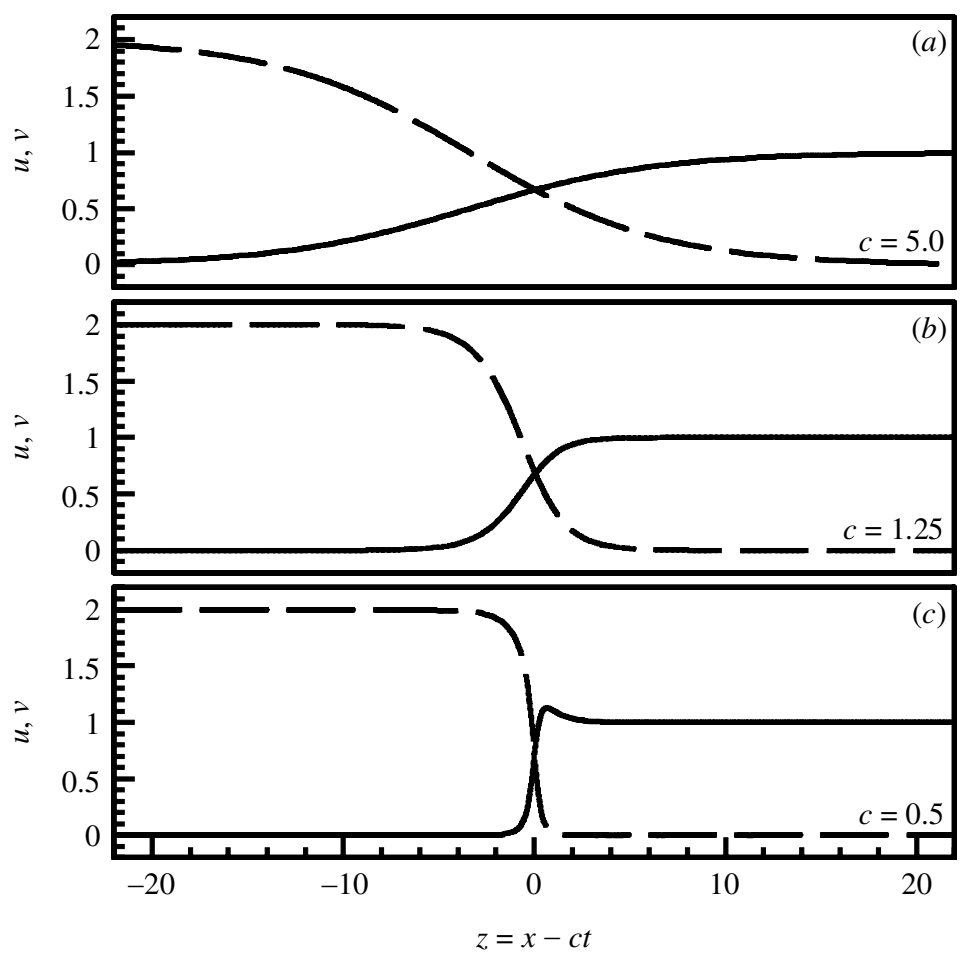

Figure 3. The form of wavefront solutions for $u(-)$ and $v(---)$, with $\gamma=2$ and with wave speeds $(a) c=5,(b) c=1.25,(c) c=0.5$. As $c$ decreases, the wave becomes steeper. In $(a)$ and $(b), c>(\gamma-1) / \sqrt{ } \gamma$, so that the $u$ wave is monotonic, but, in $(c), u$ increases above 1 and has a local maximum. The wave profiles were determined by numerical solutions of the model equations (1.1) subject to (2.1), as described in the legend to figure 1, with $(a) \xi_{u}=\xi_{v}=0.1$; (b) $\xi_{u}=\xi_{v}=0.8 ;(c) \xi_{u}=\xi_{v}=2.0$.

equation. This involves rescaling the travelling-wave coordinate by writing $\zeta=z / c$, in terms of which the travelling-wave equations (2.2) are

$$
\begin{aligned}
& \frac{1}{c^{2}} \frac{\mathrm{d}^{2} N}{\mathrm{~d} \zeta^{2}}+\frac{\mathrm{d} N}{\mathrm{~d} \zeta}-N(1+N)+(\gamma-1) V=0, \\
& \frac{1}{c^{2}} \frac{\mathrm{d}}{\mathrm{d} \zeta}\left[\left(\frac{V}{1+N}\right) \frac{\mathrm{d} N}{\mathrm{~d} \zeta}\right]+\frac{\mathrm{d} V}{\mathrm{~d} \zeta}+V(\gamma-1-N)=0 .
\end{aligned}
$$

Canosa's (1973) approach is to look for solutions as a regular perturbation expansion in $1 / c^{2}$. This is appropriate even though the $1 / c^{2}$ factors multiply the highest derivatives, because the kinetic terms are zero at both boundaries $( \pm \infty)$. Following this approach, I obtain a leading-order approximation by neglecting the terms containing factors of $1 / c$, giving two coupled first-order equations. Remarkably, these can be solved exactly by observing that

$$
\frac{\mathrm{d}}{\mathrm{d} \zeta}\left(\frac{V}{N}\right)=(\gamma-1)\left(\frac{V}{N}\right)^{2}-\gamma\left(\frac{V}{N}\right) .
$$


Solving (3.2) by separation of variables gives

$$
\frac{V}{N}=\frac{\gamma}{\gamma-1+A \exp (\gamma \zeta)}
$$

where $A \geqslant 0$ is a constant of integration. Substituting this back into (3.1) with the $\left(1 / c^{2}\right)$ terms neglected then gives

$$
\frac{\mathrm{d} V}{\mathrm{~d} \zeta}=V\left[V \frac{V}{N}(\zeta)-\gamma+1\right]
$$

This equation cannot be solved directly, but substituting $w=(\gamma-1) \zeta+\log V$ gives a separable equation for $w(\zeta)$. This leads to the solutions

$$
\begin{aligned}
& V=\gamma[B \exp \{(\gamma-1) \zeta\}-A \exp \{\gamma \zeta\}+1]^{-1}, \\
& N=[\gamma-1+A \exp \{\gamma \zeta\}][B \exp \{(\gamma-1) \zeta\}-A \exp \{\gamma \zeta\}+1]^{-1} .
\end{aligned}
$$

Since $A \geqslant 0$, the only case giving a positive solution for $N$ is $A=0$. Thus, the ratio $V / N$ is constant, and the leading-order wavefront solutions of (3.1) when $c$ is large are

$$
\begin{aligned}
N(z)=\frac{\gamma-1}{1+B \exp \{z(\gamma-1) / c\}} \quad \text { and } \quad V(z) & =\frac{\gamma}{1+B \exp \{z(\gamma-1) / c\}} \\
\Rightarrow \quad U(z) & =\frac{1}{1+B \exp \{-z(\gamma-1) / c\}} .
\end{aligned}
$$

Here, the constant $B$ is arbitrary and corresponds to a translation in the wave coordinate $z$. Comparison of this solution with numerical simulations of (1.1) subject to $(2.1)$ shows very good agreement provided $\xi_{v}$ is significantly less than $(\gamma-1)$, so that the wave speed $c$ is significantly greater than 1 (illustrated in figure 4).

Higher-order corrections to this approximate wave form can be calculated in a similar manner, but the leading-order form is sufficient to give a good qualitative understanding of the solution. In particular, it shows that both $U$ and $V / \gamma$ depend on parameters and on $z$ only through the grouping $z(\gamma-1) / c$.

\section{Minimum wave speed}

When the wave speed $c$ is large, the travelling-wave solutions of (1.1) have strong similarities with those of standard reaction-diffusion equations. Indeed, the leadingorder solution (3.3) obtained above would be the same if the motility terms in (1.1) were replaced by linear diffusion. However, as $c$ is decreased, new features emerge. I have already discussed the appearance of a local maximum in the $U$ wave, which appears as $c$ is decreased through $(\gamma-1) / \sqrt{ } \gamma$, due to the different decay rates of $V$ and $N$ ahead of the wave. This corresponds to $\xi_{v}$, the decay rate of $v$ in initial conditions (2.1), being increased through $\sqrt{ } \gamma$. As $\xi_{v}$ is increased further, the speed of the resulting wavefronts decreases, but eventually plateaus (see figure 2), suggesting that there may be a minimum possible wave speed. The existence of such a minimum speed is in itself a very familiar one in reaction-diffusion equations, having been established in the 1930s for the Fisher equation (Fisher 1937; Kolmogoroff et al. 1937), and more recently for some systems of equations (see, for example, Dunbar 


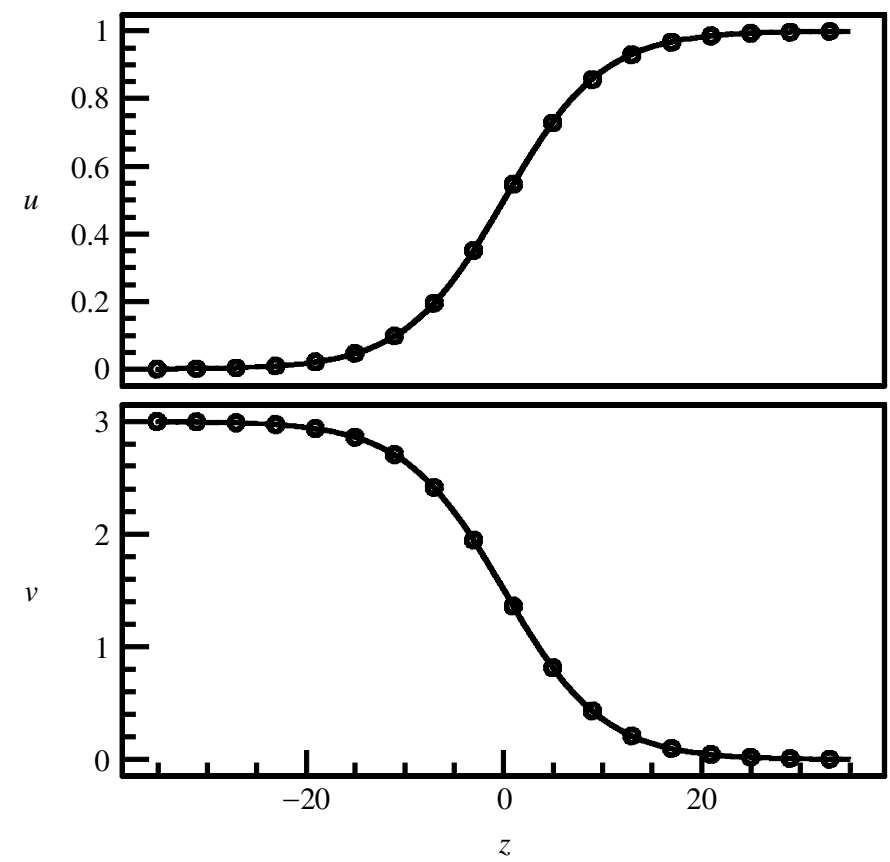

Figure 4. A comparison of the leading-order approximation (3.3) of the travelling-wave form (o) with a wave calculated by numerical solution of (1.1) subject to (2.1) (- - . The comparison is extremely good provided that the wave speed $c$ is significantly greater than 1 , as in this case $(c=10)$. The parameter values are $\gamma=3$ and $\xi_{v}=0.2$.

1984; Billingham \& Needham 1991). However, in these cases the minimum speed corresponds to a change from real to complex in the eigenvalues of the travellingwave ODEs at the equilibrium ahead of the wave; there is no such transition for (2.2). I will show that, in fact, the minimum speed arises as a result of quite new behaviour in the travelling-wave equations.

The key to understanding the minimum speed lies in the choice of travelling-wave variables. Thus far, I have been working with $V$ and $N \equiv U+V-1$. I now re-formulate the equations in terms of $N$ and $Y \equiv V /(U+V) \equiv V /(1+N)$. Substituting for $V$ in (2.2) and simplifying gives

$$
\begin{gathered}
N^{\prime \prime}+c N^{\prime}+(1+N)[(\gamma-1) Y-N]=0, \\
Y^{\prime}\left[c+N^{\prime} /(1+N)\right]=-(\gamma-1) Y(1-Y) ;
\end{gathered}
$$

recall that the prime denotes $\mathrm{d} / \mathrm{d} z$. Henceforth, I write $\Omega(z ; c) \equiv-N^{\prime} /(1+N)$ for notational ease; $\Omega$ has the qualitative form illustrated in figure 5 .

Although the shape of $N$ and, thus, the value of $\Omega_{\max } \equiv \max _{z} \Omega(z ; c)$ do depend on $c$, numerical evidence suggests that this dependence is relatively slight when $c$ is small, in keeping with the linear analysis discussed in $\S 2$. Therefore, one anticipates that as $c$ is decreased, there will be a critical value at which $c=\Omega_{\max }$, at which point the equations (4.1) become singular. This suggests that the condition $c=\Omega_{\max }$ may determine the minimum wave speed, and this is confirmed by plotting $Y$, calculated from numerical simulations of the travelling-wave solutions, as $\xi_{v}$ is increased. The qualitative form of $Y(z)$ is a monotonically decreasing transition from $Y(-\infty)=1$ 


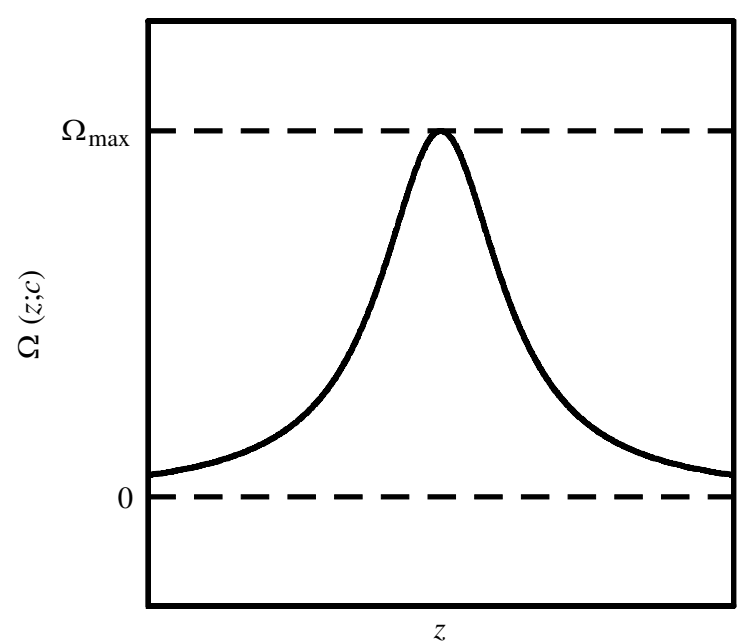

Figure 5. An illustration of the qualitative form of the expression $\Omega(z ; c) \equiv-N^{\prime} /(1+N)$ for a travelling-wave solution $N(z)$.

to $Y(+\infty)=0$. As $\xi_{v}$ is increased, this transition becomes gradually steeper, until is has the approximate form of a step function, exactly as one would expect from $(4.1 b)$ as $c+\Omega_{\max }$ approaches zero.

Having shown why there is a minimum speed for travelling waves, I now consider its value, and in particular how this depends on the parameter $\gamma$. In the limiting case as $c$ tends to its minimum value, $c_{\min }$ say, $Y(z)$ has the form of a step function; a jump occurs, at $z=0$ say, from $Y=1(z<0)$ to $Y=0(z>0)$. Therefore, to find $c_{\text {min }}$, the equation

$$
c=\Omega(z=0 ; c) \equiv \frac{-N^{\prime}(z=0)}{1+N(z=0)}
$$

must be solved, with $N(z)$ a solution of

$$
\begin{aligned}
N^{\prime \prime}+c N^{\prime} & =(1+N)[N-(\gamma-1) Y], \\
Y & = \begin{cases}1, & z<0, \\
0, & z>0 .\end{cases}
\end{aligned}
$$

Here, the end conditions are

$$
N(-\infty)=\gamma-1, \quad N(+\infty)=0,
$$

with continuity and smoothness at $z=0$ :

$$
N\left(z=0^{-}\right)=N\left(z=0^{+}\right), \quad N^{\prime}\left(z=0^{-}\right)=N^{\prime}\left(z=0^{+}\right) .
$$

For notational simplicity, I denote the common value of $N\left(z=0^{ \pm}\right)$by $N^{*}$.

Solution of (4.2), (4.3), (4.4) for $c_{\min }$ is a difficult problem, and I begin by discussing numerical methods. The natural approach to numerical solution of (4.3) subject to (4.4) would be to solve separately on $z<0$ and $z>0$, using a shooting method for each. This is a feasible approach, but a laborious one, since it requires varying the value of $N\left(z=0^{-}\right)=N\left(z=0^{+}\right)$until the values of $N^{\prime}\left(z=0^{-}\right)$and $N^{\prime}\left(z=0^{+}\right)$ 
given by the shooting method are the same. A more efficient approach is possible if one re-formulates the equations with $N$ as independent variable: this is possible since we anticipate that $N$ monotonically decreases with $z$. The new equations are

$$
\begin{aligned}
\frac{\mathrm{d} W}{\mathrm{~d} N} & =-c+\frac{(1+N)[N-(\gamma-1) Y]}{W}, \\
\frac{\mathrm{d} z}{\mathrm{~d} N} & =\frac{1}{W},
\end{aligned}
$$

where $Y$ is as in $(4.3 b)$ and $W \equiv N^{\prime}$. I solved these equations numerically as initialvalue problems, integrating with $N$ increasing from $N=\varepsilon_{0}$ with $Y \equiv 0$, and with $N$ decreasing from $\gamma-1-\varepsilon_{0}$ with $Y \equiv 1$. Here, $\varepsilon_{0}$ is a small parameter whose value can be successively decreased to give greater accuracy; the two initial values of $W$ are determined from the eigenvectors at the corresponding equilibria, and the initial values of $z$ are arbitrary. Once both initial-value problems have been solved, a simple comparison of the solutions reveals the value of $N$ at which $W$ is the same in the two solutions; this is straightforward, with $N$ taken as dependent variable, since there is then an evenly spaced grid of $N$-values in the numerical solution, and linear interpolation between grid points is all that is required. The solution is then obtained by translation by appropriate amounts in $z$ in the two solutions, so that the point of intersection is at $z=0$. With an efficient method of solving (4.3) subject to (4.4), $c_{\min }$ can easily be found numerically by solving (4.2) using any standard method for a nonlinear algebraic equation. A typical wave solution at $c=c_{\min }$ is shown in figure 6 .

Exact analytical calculation of $c_{\min }$ would require an exact solution of (4.3), which does not seem possible. However, a method of calculating an analytical approximation is suggested by a plot of the left- and right-hand sides of (4.2) against $c$, as illustrated in figure 7 .

This reveals that $\Omega(z=0 ; c)$ varies much more gradually with $c$ than the lefthand side $c$. Thus, one expects that a good approximation to $c_{\text {min }}$ will be given by $\Omega(z=0 ; c=0)$, which can be calculated exactly. Consider first $(4.3 a)$ with $Y \equiv 0$ and $c=0$,

$$
N^{\prime \prime}=N(1+N)
$$

which is subject to

$$
N(+\infty)=0
$$

Multiplying (4.6) through by $N^{\prime}$ enables exact integration, and, with condition (4.7), this gives

$$
N^{\prime}=-N \sqrt{1+\frac{2}{3} N}
$$

This can be solved by separation of variables, giving

$$
N=\frac{3}{2}\left(\left[\frac{1+A_{1} \mathrm{e}^{-z}}{1-A_{1} \mathrm{e}^{-z}}\right]^{2}-1\right),
$$

where $A_{1}$ is a constant of integration. Similarly, one can solve $(4.3 a)$ with $Y \equiv 1$ and $c=0$, subject to $N(-\infty)=0$. In fact, a separate solution is not even required, since substituting

$$
\hat{N}=(N-\gamma+1) / \gamma, \quad \hat{z}=-z \sqrt{ } \gamma
$$



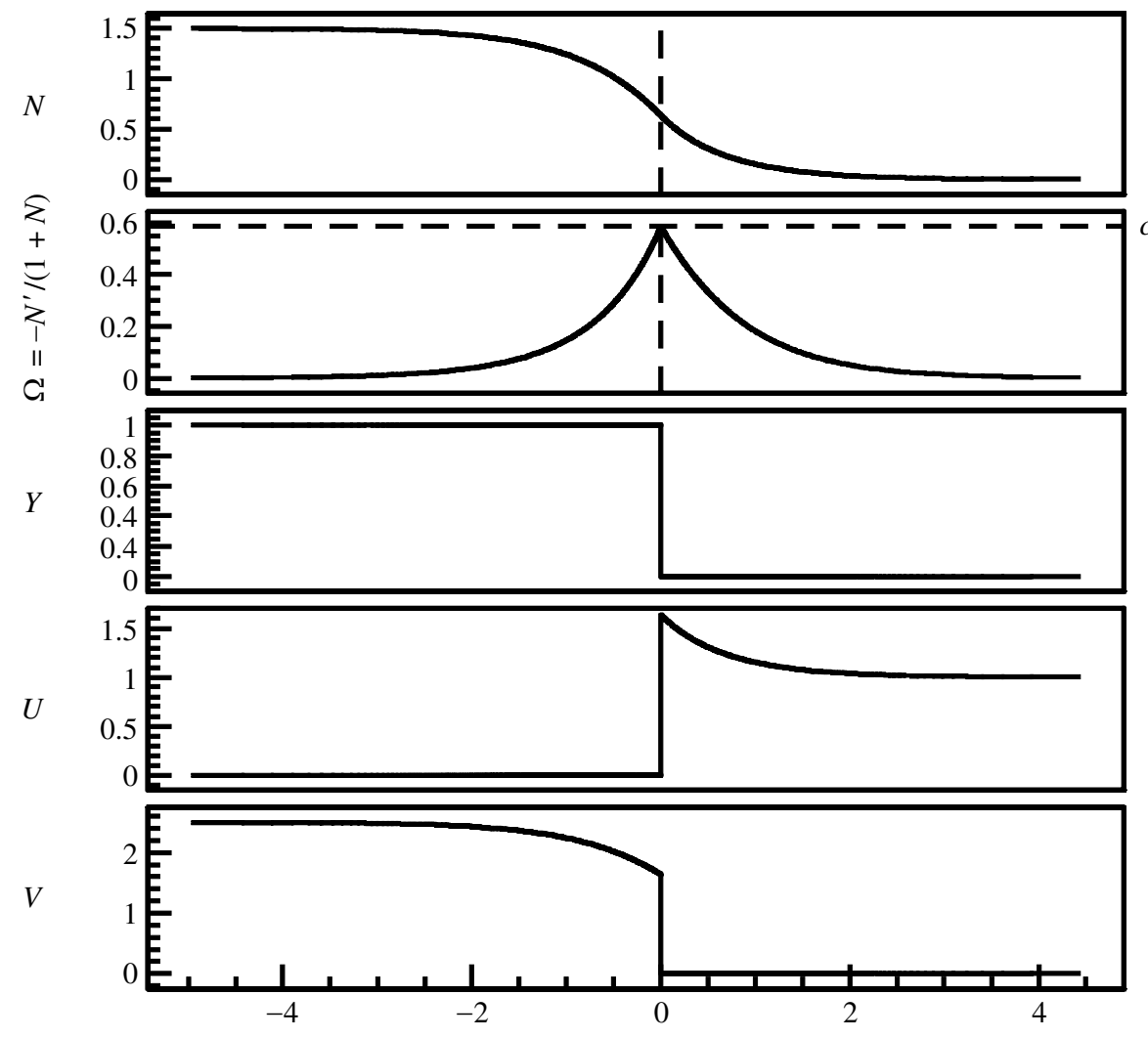

$z$

Figure 6. An illustration of the travelling-wave solution at the critical minimum wave speed $c_{\mathrm{m} \text { in }}$. The point $z=0$ is illustrated by the vertical dashed line: $Y(z) \equiv U /(U+V)$ jumps from 1 to 0 at this point, with corresponding jumps in $U$ and $V$. The solution for $N$ is smooth at $z=0$ but with a discontinuity in its second derivative; $\Omega$ has a cusp at $z=0$ with the maximum value $c$ $\left(=c_{\mathrm{m} \text { in }}\right)$. The solution is calculated by numerical solution of (4.3) subject to (4.4), as described in the main text. The parameter $\gamma$ is equal to 2.5 ; this implies $c_{\mathrm{m} \text { in }} \approx 0.5863$.

gives (4.6) and (4.7) to be solved for $\hat{N}(\hat{z})$. Thus, the solution for $N(z)$ for $z<0$ is

$$
N=\gamma-1+\frac{3}{2} \gamma\left(\left[\frac{1+A_{2} \mathrm{e}^{+z \sqrt{ } \gamma}}{1-A_{2} \mathrm{e}^{+z \sqrt{ } \gamma}}\right]^{2}-1\right) .
$$

The solution for $N(z ; c=0)$ is given by (4.8) for $z>0$, and by (4.10) for $z<0$. The conditions $(4.4 b)$ at $z=0$ enable calculation of the constants $A_{1}$ and $A_{2}$, and $\Omega(z ; c=0)$ can then be found directly as $-N^{\prime}(z ; c=0) /[1+N(z ; c=0)]$. This gives a first approximation to $c_{\min }$ as

$$
\begin{aligned}
& c_{\min } \approx \Omega(z=0 ; c=0)=\frac{\xi \sqrt{1+\frac{2}{3} \xi}}{1+\xi}, \\
& \text { where } \xi=N(z=0 ; c=0)=\sqrt{\left(\gamma^{2}+\gamma+1\right) / 3}-1 .
\end{aligned}
$$




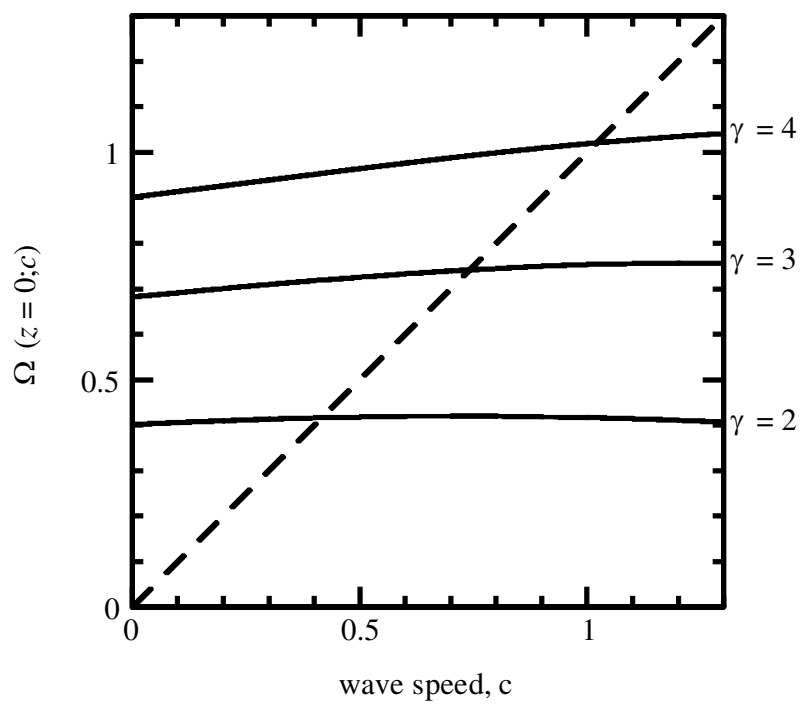

Figure 7. A plot of the left-hand side $c(---)$ and right-hand side $\Omega(z=0 ; c)$ (- $(-)$ of (4.2) against $c$, for three different values of the parameter $\gamma$. The minimum possible wave speed $c_{\mathrm{m} i n}$ is given by the intersection of these lines. The values of $\Omega(z=0 ; c)$ are given by numerical solution of (4.3) subject to (4.4), as described in the main text.

This approximation is compared with numerically calculated values of $c_{\text {min }}$ in figure 8 . The comparison is very good at small values of $\gamma$, but becomes worse as $\gamma$ is increased.

A more accurate approximation to the minimum speed can be found by replacing the right-hand side of (4.2) by a power series approximation for $\Omega(z=0 ; c)$ about $c=0$. The approximation (4.11) corresponds to taking just the constant term in this power series, and I will show that it can be improved dramatically by also including the linear term in $c$. Looking for a power series approximation for $N(z ; c)$ gives

$$
N=N_{0}+c N_{1}+c^{2} N_{2}+\cdots,
$$

where $N_{0}$ is given by (4.8) when $z>0$ and (4.10) when $z<0$. In the region $z>0$, $N_{1}$ satisfies

$$
N_{1}^{\prime \prime}+N_{0}^{\prime}-\left(1+2 N_{0}\right) N_{1}=0,
$$

subject to $N_{1}(+\infty)=0$. This can be solved by treating $N_{1}$ as a function of $N_{0}$, which is appropriate since $N_{0}(z)$ is monotonic. Then

$$
\left(N_{0}^{2}+\frac{2}{3} N_{0}^{3}\right) \frac{\mathrm{d}^{2} N_{1}}{\mathrm{~d} N_{0}^{2}}+\left(N_{0}+N_{0}^{2}\right) \frac{\mathrm{d} N_{1}}{\mathrm{~d} N_{0}}-\left(1+2 N_{0}\right) N_{1}=N_{0} \sqrt{1+\frac{2}{3} N_{0}},
$$

i.e.

$$
\begin{aligned}
& \frac{\mathrm{d}}{\mathrm{d} N_{0}}\left[\left(N_{0}^{2}+\frac{2}{3} N_{0}^{3}\right) \frac{\mathrm{d} N_{1}}{\mathrm{~d} N_{0}}-\left(N_{0}+N_{0}^{2}\right) N_{1}\right]=N_{0} \sqrt{1+\frac{2}{3} N_{0}} \\
\Rightarrow \quad \frac{\mathrm{d} N_{1}}{\mathrm{~d} N_{0}} & =\mathcal{R}\left(N_{0}\right) N_{1}+\mathcal{S}\left(N_{0}\right),
\end{aligned}
$$




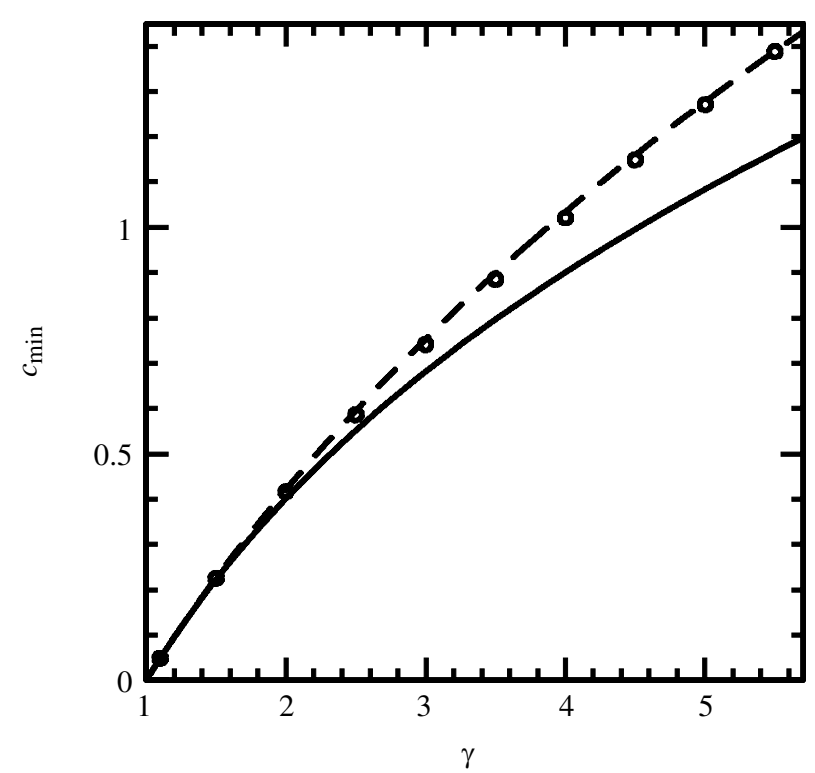

Figure 8. A plot of numerically calculated values of $c_{\min }(\circ)$, with zeroth-order (- - and first-order (- - ) approximations, given by (4.11) and (4.17), respectively. The numerically calculated values are given by solving (4.2) using numerical solutions of (4.3) subject to (4.4), as described in the main text.

where

$$
\mathcal{R}\left(N_{0}\right)=\frac{1+N_{0}}{N_{0}\left(1+\frac{2}{3} N_{0}\right)}
$$

and

$$
\mathcal{S}\left(N_{0}\right)=\left(3 / 5 N_{0}^{2}\right)\left[\left(N_{0}-1\right)\left(1+\frac{2}{3} N_{0}\right)^{1 / 2}+\left(1+\frac{2}{3} N_{0}\right)^{-1}\right] .
$$

This can be integrated further to obtain $N_{1}$ explicitly, but (4.13) is sufficient for calculation of $c_{\min }$. The expression corresponding to (4.13) for $z<0$ can be determined directly: the substitutions (4.9) together with $\hat{c}=-c / \sqrt{ } \gamma$ convert the problem for $z<0$ to that for $z>0$. This gives

$$
\frac{\mathrm{d} N_{1}}{\mathrm{~d} N_{0}}=\frac{-1}{\sqrt{ } \gamma}\left[-\mathcal{R}\left(\frac{N_{0}-\gamma+1}{\gamma}\right) \frac{N_{1}}{\sqrt{ } \gamma}+\mathcal{S}\left(\frac{N_{0}-\gamma+1}{\gamma}\right)\right],
$$

for $z<0$. Since $N_{1}$ and $N_{0}$ are both continuous and smooth at $z=0$, the righthand sides of (4.13) and (4.14) can be set equal and solved for the common value of $N_{1}(z=0)$, giving

$$
N_{1}(z=0)=\Upsilon \equiv\left(\gamma^{-1 / 2} \mathcal{S}\left(\frac{\xi-\gamma+1}{\gamma}\right)+\mathcal{S}(\xi)\right) /\left(\gamma^{-1} \mathcal{R}\left(\frac{\xi-\gamma+1}{\gamma}\right)-\mathcal{R}(\xi)\right)
$$

Here $\xi$ is the value of $N_{0}(z=0)$, defined in (4.11). 
Turning now to $\Omega(z ; c) \equiv-N^{\prime}(z ; c) /[1+N(z ; c)]$, expanding as a power series in $c$ gives

$$
\Omega(z=0 ; c)=\Omega(z=0 ; c=0)+c \Omega(z=0 ; c=0)\left[\frac{\mathrm{d} N_{1}}{\mathrm{~d} N_{0}}-\frac{N_{1}}{1+N_{0}}\right]+\cdots .
$$

The value of $\Omega(z=0 ; c=0)$ is given in (4.11). Substituting the first two terms of this expansion into (4.2), the equation for $c_{\mathrm{min}}$, gives the improved approximation

$$
c_{\min } \approx \frac{\xi \sqrt{1+2 \xi / 3}}{1+\xi-\xi \sqrt{1+2 \xi / 3}[\mathcal{R}(\xi) \Upsilon+\mathcal{S}(\xi)-\Upsilon /(1+\xi)]}
$$

recall that $\xi$ is defined in (4.11), $\mathcal{R}(\cdot)$ and $\mathcal{S}(\cdot)$ are defined in (4.13), and $\Upsilon$ is defined in (4.15). Strictly, (4.17) is valid as a first-order approximation for small $c_{\min }$, i.e. for $\gamma$ close to 1 . However, in practice it is extremely close to numerically calculated values of $c_{\min }$ for a wide range of $\gamma$, as illustrated in figure 8 . The reason for the approximation being so good is that $\Omega(z=0 ; c)$ is approximately linear as a function of $c$, as illustrated in figure 7, so that higher-order terms in the expansion (4.16) are extremely small.

\section{Waveform close to the minimum speed}

At the minimum speed itself, $c=c_{\text {min }}$, the form of the travelling wavefront has been established in the above discussion: $Y(z)$ is a step function with a jump from $Y=1$ to $Y=0$ at $z=0$, and $N(z)$ has a monotonically decreasing form, with a discontinuity in its second derivative at $z=0$. The form of the $N$ wave is illustrated numerically in figure 6 above, and an analytical approximation for small $c_{\text {min }}$ (i.e. for $\gamma$ close to 1 ) is given by (4.8) and (4.10). In this section, I use perturbation theory to investigate the form of waves with speeds slightly above the minimum, with the objective of understanding the way in which $Y(z)$ approaches a step function as $c$ is decreased. Absence of an analytical form for the minimum speed wave prevents calculation of a full matched expansion, but an approximation to $Y$ close to the minimum speed can be found.

I consider the travelling wave equations (4.1) for wave speed $c=c_{\min }+\epsilon$, with $\epsilon \ll 1$. It is most convenient to rewrite $(4.1 a)$ as two first-order equations, using the variables $N(z)$ and $\Omega(z) \equiv-N^{\prime}(z) /(1+N(z))$, giving

$$
\begin{aligned}
& \frac{\mathrm{d} N}{\mathrm{~d} z}=-\Omega(1+N), \\
& \frac{\mathrm{d} \Omega}{\mathrm{d} z}=\Omega\left(\Omega-c_{\min }-\epsilon\right)+(\gamma-1) Y-N, \\
& \frac{\mathrm{d} Y}{\mathrm{~d} z}=\frac{-(\gamma-1) Y(1-Y)}{c_{\min }+\epsilon-\Omega} .
\end{aligned}
$$

I have shown that $Y$ approaches a step function as $\epsilon \rightarrow 0$; as above, I take the jump to occur at $z=0$. Then (5.1) are the outer equations, valid for small $\epsilon$ away from $z=0$. The two parts of the outer solution will clearly be joined by a transition layer centred at $z=0$, with the rescaled wave coordinate $\tilde{z}=z / \tilde{\mu}_{z}(\epsilon)$, where $\tilde{\mu}_{z}(\epsilon)$ is $o(1)$ 
as $\epsilon \rightarrow 0$. Denoting transition-layer solutions by tildes, the inner equations are, thus,

$$
\begin{aligned}
& \frac{\mathrm{d} \tilde{N}}{\mathrm{~d} \tilde{z}}=-\tilde{\mu}_{z}(\epsilon) \tilde{\Omega}(1+\tilde{N}), \\
& \frac{\mathrm{d} \tilde{\Omega}}{\mathrm{d} \tilde{z}}=\tilde{\mu}_{z}(\epsilon)\left[\tilde{\Omega}\left(\tilde{\Omega}-c_{\min }-\epsilon\right)+(\gamma-1) \tilde{Y}-\tilde{N}\right], \\
& \frac{\mathrm{d} \tilde{Y}}{\mathrm{~d} \tilde{z}}=\frac{-\tilde{\mu}_{z}(\epsilon)(\gamma-1) \tilde{Y}(1-\tilde{Y})}{c_{\min }+\epsilon-\tilde{\Omega}} .
\end{aligned}
$$

I look for a series solution of these inner equations:

$$
\begin{aligned}
& \tilde{N}(\tilde{z})=\tilde{N}_{0}(\tilde{z})+\tilde{\mu}_{N}(\epsilon) \tilde{N}_{1}(\tilde{z})+\cdots, \\
& \tilde{\Omega}(\tilde{z})=\tilde{\Omega}_{0}(\tilde{z})+\tilde{\mu}_{\Omega}(\epsilon) \tilde{\Omega}_{1}(\tilde{z})+\cdots, \\
& \tilde{Y}(\tilde{z})=\tilde{Y}_{0}(\tilde{z})+\tilde{\mu}_{Y}(\epsilon) \tilde{Y}_{1}(\tilde{z})+\cdots,
\end{aligned}
$$

where the functions $\tilde{\mu}_{N}, \tilde{\mu}_{\Omega}$ and $\tilde{\mu}_{Y}$ are $o(1)$ as $\epsilon \rightarrow 0$. Substituting these expansions into (5.2) shows that $N_{0}$ is constant, and this clearly must be equal to $N^{*}$, the leadingorder value of the outer solution on both sides of the transition layer. Similarly, $\tilde{\Omega}_{0} \equiv c_{\min }$. This implies that $\tilde{Y}_{0}$ satisfies

$$
\frac{\mathrm{d} \tilde{Y}_{0}}{\mathrm{~d} \tilde{z}}=\frac{-\tilde{\mu}_{z}(\epsilon)(\gamma-1) \tilde{Y}_{0}\left(1-\tilde{Y}_{0}\right)}{\epsilon-\tilde{\mu}_{\Omega}(\epsilon) \tilde{\Omega}_{1}} .
$$

The distinguished limit is, thus, given by $\tilde{\mu}_{z}(\epsilon)=\tilde{\mu}_{\Omega}(\epsilon)=\epsilon$, in which case

$$
\frac{\mathrm{d} \tilde{Y}_{0}}{\mathrm{~d} \tilde{z}}=\frac{-(\gamma-1) \tilde{Y}_{0}\left(1-\tilde{Y}_{0}\right)}{1-\tilde{\Omega}_{1}}
$$

This equation must be solved together with

$$
\mathrm{d} \tilde{\Omega}_{1} / \mathrm{d} \tilde{z}=(\gamma-1) \tilde{Y}_{0}-N^{*},
$$

which is given by substituting (5.3) into (5.2) and equating terms of order $\epsilon$. Since we expect $\tilde{Y}_{0}(\tilde{z})$ to be monotonic, we can treat $\tilde{\Omega}_{1}$ as a function of $\tilde{Y}_{0}$, and dividing (5.5) by (5.4) gives a separable ODE, with solution

$$
\tilde{\Omega}_{1}=1-\frac{K}{\tilde{Y}_{0}^{\nu}\left(1-\tilde{Y}_{0}\right)^{(1-\nu)}} .
$$

Here $K$ is a constant of integration, and $\nu=N^{*} /(\gamma-1)$. This is a key parameter grouping; numerical calculation of the leading-order outer solution, as described in $\S 4$, enables calculation of $N^{*}$, and this shows that $\nu$ decreases from $\frac{1}{2}$ as $\gamma$ increases from 1 (figure $9 a$ ).

Substituting (5.6) back into (5.4) shows that $\tilde{Y}_{0}$ is defined implicitly by the formula

$$
\tilde{z}=\frac{K}{(\gamma-1) \nu(1-\nu)}\left[2 \nu-1+\frac{1-\tilde{Y}_{0}-\nu}{\tilde{Y}_{0}^{\nu}\left(1-\tilde{Y}_{0}\right)^{1-\nu}}\right] .
$$

Derivation of this formula is discussed in more detail in the appendix; there is a constant of integration that corresponds to an order- $\epsilon$ translation of the travelling wave, which I have chosen to give $\tilde{Y}_{0}(\tilde{z}=0)=\frac{1}{2}$. 

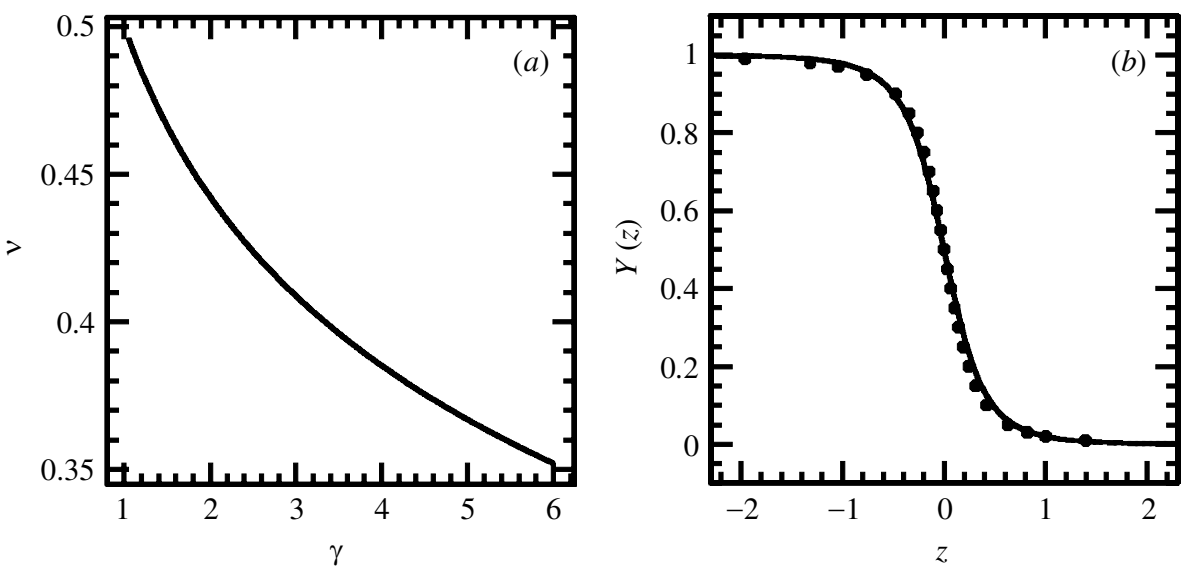

Figure 9. (a) A plot of numerically calculated values of $\nu=N^{*} /(\gamma-1)$, given by solving (4.2) using numerical solutions of (4.3) subject to (4.4), as described in $\S 4$ of the main text. Solutions for large values of $\gamma$ suggest that $\nu \rightarrow 0$ as $\gamma \rightarrow \infty$. (b) A comparison between the approximation $((5.7)$, with $\tilde{z}=z / \epsilon)$ for $Y(z)(\bullet)$ and a numerically calculated wave form (- $(-)$, found via numerical solution of the model PDEs (1.1). The comparison is good and improves even further as $\epsilon=c-c_{\mathrm{m}}$ in is reduced. The parameters in this case are $\gamma=2$ and $\xi_{v}=2$, giving a wave speed $c=2$, while $c_{\mathrm{min}} \approx 0.4163$. The constant $K$ is taken to be 1 .

The condition $K>0$ must hold in order that $\tilde{Y}_{0}(-\infty)=1$ and $\tilde{Y}_{0}(+\infty)=0$. The value of $K$ is determined by matching this inner solution with higher-order terms in the outer solution, and, thus, $K$ cannot be determined analytically without an exact solution for the leading-order solutions for $N$ and $\Omega$. Comparison between (5.7) and numerically calculated waves indicates that $K \approx 1$ : one such comparison is illustrated in figure $9 \mathrm{~b}$. The key implication of the calculation is the form of $Y$ for $c$ close to $c_{\min }$ : because of the simple form of the leading-order outer solution, (5.7) is a leading-order approximation to the solution for $Y$ (with $\tilde{z}=z / \epsilon$ ). Note, in particular, that this approximation approaches 0 and 1 (as $z \rightarrow+\infty$ and $z \rightarrow-\infty$, respectively) algebraically, but at different rates for the two limits.

\section{Discussion}

In this paper, I have presented a simple way of incorporating contact inhibition of cell migration into a mathematical model for interacting cell populations. I have considered this in the particular context of two competing populations, one of which has a proliferative advantage over the other; this is motivated by the very early stages of solid tumour growth. The model is a very generic one, but accurately captures the basic process of a moving interface between the two cell populations without widespread mixing. I have presented a detailed study of these moving interfaces, which indicates that there are solutions for wave speeds above a critical minimum value. I have shown that the minimum speed arises via quite new behaviour in the travelling-wave equations, with the proportion of cells of each type approaching a step function as the wave speed decreases towards the minimum. Exploiting this structure, I have used singular perturbation theory to investigate the wave shape for speeds close to the minimum. 
The key results in this paper concern the speed of travelling waves, and, in particular, the value of the minimum speed $c_{\min }$, which is generated by the biologically realistic case of highly localized initial cell populations. A natural point of comparison is provided by the speeds of travelling-wave solutions of the equations with the same kinetics as (1.1), but with linear diffusion for both cell populations, namely

$$
\begin{aligned}
& \frac{\partial u}{\partial t}=\frac{\partial^{2} u}{\partial x^{2}}+u(1-u-v) \\
& \frac{\partial v}{\partial t}=\frac{\partial^{2} v}{\partial x^{2}}+v(\gamma-u-v)
\end{aligned}
$$

Numerical solutions of (6.1) on an infinite spatial domain with initial conditions (2.1) and with $\gamma>1$ evolve to travelling-wave solutions with the same qualitative form as illustrated for (1.1) in figure $1 b$ : an advancing wave of $v$ in parallel with a receding wave of $u$. Linearizing (6.1), and drawing analogy with the Fisher equation, enables the value of the wave speed to be predicted as

$$
c^{\mathrm{diff}}= \begin{cases}\xi+(\gamma-1) / \xi, & \xi \leqslant \sqrt{\gamma-1}, \\ 2 \sqrt{\gamma-1}, & \xi \geqslant \sqrt{\gamma-1}\end{cases}
$$

this is confirmed by numerical measurement of wave speed. For small values of $\xi$, $c^{\text {diff }}$ is greater than the wave speed $c$ that I have found for (1.1), by the amount $\xi$ : as expected, the inclusion of contact inhibition of migration slows down the moving interface between the cell populations. Moreover, as $\xi$ is decreased through $\sqrt{\gamma-1}$, $c^{\text {diff }}$ becomes constant at the minimum wave speed $c_{\text {min }}^{\text {diff }}=2 \sqrt{\gamma-1}$, while $c$ continues to decrease to $c_{\min }$ (figure $10 a$ ). My calculations in $\S 4$ show that $c_{\min }^{\text {diff }}$ is greater than $c_{\min }$ for all $\gamma>1$ (figure 10b). The approximation (4.17) for $c_{\min }$ derived in $\S 4$ enables a quantitative comparison, but this is algebraically rather complex. It is more instructive to consider limiting behaviour: straightforward expansion of (4.17) shows that

$$
\begin{aligned}
c_{\min } \sim \frac{1}{2}(\gamma-1), & \text { as } \gamma \rightarrow 1^{+}, \\
c_{\min } \sim\left[\frac{2^{1 / 2} 3^{-3 / 4} 5}{3-\left\{\frac{3^{-1 / 4} 10 \mathcal{S}(-1+1 / \sqrt{ } 3)+2 \sqrt{ } 6}{2 \sqrt{2} \mathcal{R}(-1+1 / \sqrt{ } 3)-3 \sqrt{ } 6}\right\}}\right] \sqrt{ } \gamma \approx 0.825 \sqrt{ } \gamma, & \text { as } \gamma \rightarrow \infty .
\end{aligned}
$$

Thus, the ratio $c_{\min }^{\mathrm{diff}} / c_{\mathrm{min}}$ decreases from infinity as $\gamma$ increases from 1 (figure 10c), approaching a limiting value of about 2.4 (approximately $2 / 0.825$ ) as $\gamma \rightarrow \infty$. Thus, my analysis shows that the use of simple linear diffusion for competing cell populations gives an overestimate of the speed of interfaces between the populations, by a factor that is at least 2.4 , and is very much larger when the competitive advantage is small.

There is a long history of work on wavefronts in competition models that are generalizations of (6.1), with both species diffusing linearly but with a more general 

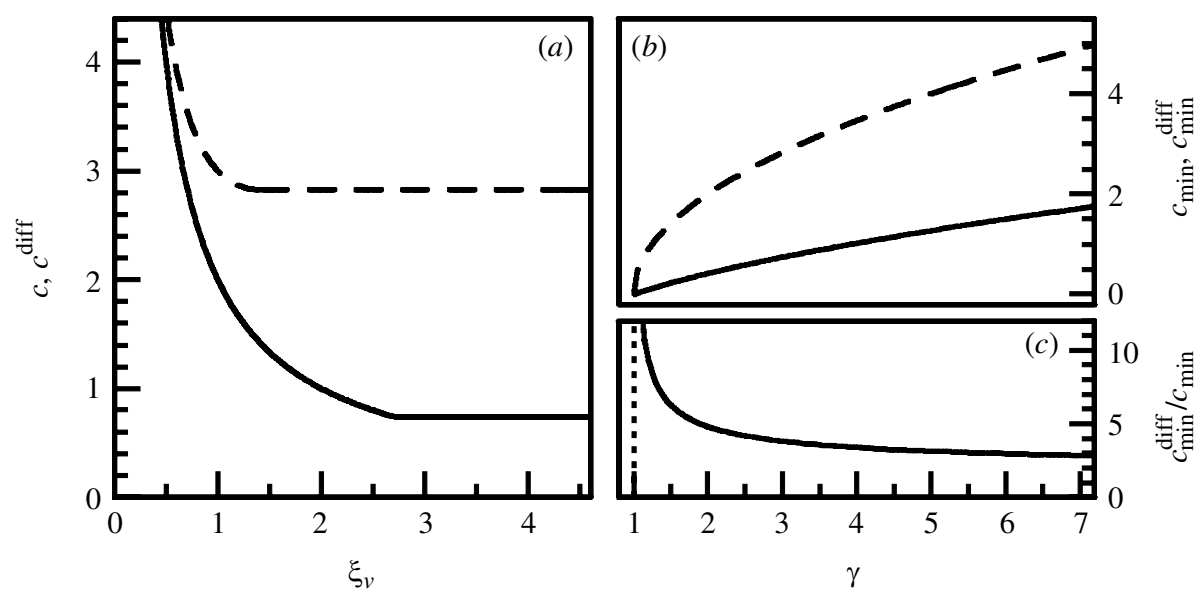

Figure 10. A comparison of wave speeds in the contact inhibition model (1.1) ( -$)$ and the model (6.1) with linearly diffusing cell populations $(---)$. (a) A plot of wave speeds $c$ and $c^{\text {diff }}$ as a function of initial decay rate $\xi_{v}$. For each $\xi_{v}$, the waves generated in the model (6.1) with linear diffusion move faster than those in (1.1): as expected, contact inhibition of migration slows down the movement of wavefronts. The difference is exactly $\xi_{v}$ for $\xi_{v}<\sqrt{\gamma-1}$, but increases for larger $\xi_{v}$, since $c$ continues to decrease once $c^{\text {diff }}$ has reached its minimum value. The value of $c^{\text {diff }}$ is calculated using (6.2); for (1.1), $c$ is given by (2.5), with the minimum wave speed found by solving (4.2) using numerical solutions of (4.3) subject to (4.4), as described in $\S 4$ of the main text. The cases shown are for $\gamma=3$. (b) A plot of the minimum wave speeds $c_{\mathrm{m}}$ in and $c_{\mathrm{min}}^{\mathrm{diff}}=2 \sqrt{\gamma-1}$ as a function of $\gamma$. The minimum speed for (1.1) is less than that for (6.1) for all $\gamma>1$. The value of $c_{\mathrm{m}}$ in is found by solving (4.2) using numerical solutions of (4.3) subject to (4.4), as described in $\S 4$ of the main text. $(c)$ A plot of the ratio $c_{\mathrm{min}}^{\mathrm{diff}} / c_{\mathrm{m} \text { in }}$ as a function of $\gamma$.

version of the Lotka-Volterra competition kinetics. The equations concerned are

$$
\begin{aligned}
& \frac{\partial u}{\partial t}=\frac{\partial^{2} u}{\partial x^{2}}+u\left(1-u-a_{1} v\right), \\
& \frac{\partial v}{\partial t}=\frac{\partial^{2} v}{\partial x^{2}}+v\left(a_{2}-a_{3} u-v\right) .
\end{aligned}
$$

The first study of wavefronts in (6.3) was that of Tang \& Fife (1980), who studied waves with $u=v=0$ ahead of the front, and $u$ and $v$ both non-zero behind; the latter type of equilibrium exists provided $a_{1} a_{3} \neq 1$. This was extended to an arbitrary number of interacting populations by Ahmad \& Lazer (1991) and to more general kinetics by van Vuuren (1995). Again in the case $a_{1} a_{3} \neq 1$, Gardner (1982) and Conley \& Gardner (1984) proved the existence of waves connecting the equilibria $(1,0)$ and $\left(0, a_{2}\right)$, with subsequent extensions by Mimura \& Fife (1986) and Kan-on (1995). This is quite different from the case considered in the present paper, because these results only apply when the two equilibria are both locally stable in the kinetic ODEs; an important challenge for future work is the investigation of such waves when the nonlinear motility terms from (1.1) are used in (6.3) rather than linear diffusion. Waves analogous to those I have studied occur for $(6.3)$ when $a_{2}>\max \left(a_{3}, 1 / a_{1}\right)$; this condition includes $(6.1)$, and ensures that $(1,0)$ is locally unstable with $\left(0, a_{2}\right)$ locally stable. Such waves were first studied numerically, with analytical solution in a special case, by Okubo et al. (1989). Existence of waves was proved by Kan-on (1997), with 
extension to the case of different diffusion coefficients considered by Hosono (1998). The work presented in the present paper raises many challenges for the extension of these various results to the new movement term reflecting contact inhibition.

\section{Appendix A.}

In this short appendix, I give some further details of the derivation of formula (5.7) for $\tilde{Y}_{0}$. Substitution of (5.6) into (5.4) gives a separable first-order ODE for $\tilde{Y}_{0}(\tilde{z})$, which implies that

$$
\frac{(\gamma-1) \tilde{z}}{K}=\mathcal{H}\left(\tilde{Y}_{0}\right)
$$

where

$$
\mathcal{H}(s)=\int_{y=s}^{y=1 / 2} \frac{\mathrm{d} y}{y^{1+\nu}(1-y)^{2-\nu}} .
$$

The upper limit of integration is arbitrary, corresponding to an $O(\epsilon)$ translation in the travelling wave; as discussed in the main text, I choose $\frac{1}{2}$ in order to give $\tilde{Y}_{0}(0)=\frac{1}{2}$. The integral defining $\mathcal{H}(\cdot)$ can be evaluated exactly by making the substitution $y=$ $\sin ^{2} \theta$, which gives

$$
\mathcal{H}(s)=2 \int_{\theta=\arcsin \sqrt{ } s}^{\theta=\pi / 4} \frac{\mathrm{d} \theta}{\sin ^{1+2 \nu} \theta \cos ^{3-2 \nu} \theta} .
$$

Integrating by parts then gives

$$
\begin{aligned}
\mathcal{H}(s) & =\left[\frac{-1}{\nu \sin ^{2 \nu} \theta \cos ^{2(1-\nu)} \theta}\right]_{\theta=\arcsin \sqrt{ } s}^{\theta=\pi / 4}+\frac{2}{\nu} \int_{\theta=\arcsin \sqrt{ } s}^{\theta=\pi / 4} \sec ^{2} \theta \tan ^{1-2 \nu} \theta \mathrm{d} \theta \\
& =\left[\frac{-1}{\nu \sin ^{2 \nu} \theta \cos ^{2(1-\nu)} \theta}+\frac{\tan ^{2(1-\nu)} \theta}{\nu(1-\nu)}\right]_{\theta=\arcsin \sqrt{ } s}^{\theta=\pi / 4} \\
& =\frac{1}{\nu(1-\nu)}\left[2 \nu-1+\frac{1-s-\nu}{s^{\nu}(1-s)^{1-\nu}}\right] .
\end{aligned}
$$

The formula (5.7) follows immediately from this.

\section{References}

Abercrombie, M. 1970 Contact inhibition in tissue culture. In vitro 6, 128-140.

Ahmad, S. \& Lazer, A. C. 1991 An elementary approach to travelling front solutions to a system of $N$-competition-diffusion equations. Nonlinear Analysis TMA 16, 893-901.

Billingham, J. \& Needham, D. J. 1991 The development of travelling waves in quadratic and cubic autocatalysis with unequal diffusion rates. I. Permanent form travelling waves. Phil. Trans. R. Soc. Lond. A 334, 1-24.

Brown, P. N., Byrne, G. D. \& Hindmarsh, A. C. 1989 VODE, a variable coefficient ODE solver. SIAM J. Sci. Stat. Comput. 10, 1038-1051.

Byrne, H. M. \& Chaplain, M. A. J. 1997 Free boundary value problems associated with the growth and development of multicellular spheroids. Eur. J. Appl. Math. 8, 639-658. 
Canosa, J. 1973 On a nonlinear diffusion equation describing population growth. IBM J. Res. Dev. 17, 307-313.

Chaplain, M. A. J. \& Stuart, A. M. 1991 A mathematical model for the diffusion of tumor angiogenesis factor into the surrounding host tissue. IMA J. Math. Appl. Med. Biol. 8, 191220.

Chaplain, M. A. J. \& Stuart, A. M. 1993 A model mechanism for the chemotactic response of endothelial cells to tumor angiogenesis factor. IMA J. Math. Appl. Med. Biol. 10, 149-168.

Conley, C. \& Gardner, R. 1984 An application of the generalised Morse index to travelling wave solutions of a competitive reaction-diffusion model. Indiana Univ. Math. J. 33, 319-343.

Dale, P. D., Sherratt, J. A. \& Maini, P. K. 1997 The role of fibroblast migration in collagen fibre formation during foetal and adult dermal wound healing. Bull. Math. Biol. 59, 1077-1100.

Dunbar, S. R. 1984 Traveling wave solutions of diffusive Lotka-Volterra equations: a heteroclinic connection in $R^{4}$. Trans. Am. Math. Soc. 286, 557-594.

Fisher, R. A. 1937 The wave of advance of advantageous genes. Ann. Eugenics 7, 353-369.

Freidlin, M. I. 1983 On wavefront propagation in multicomponent media. Trans. Am. Math. Soc. 276, 181-191. (See also the erratum: Trans. Am. Math. Soc. 289, 429.)

Gaffney, E. A., Maini, P. K., Sherratt, J. A. \& Tuft, S. 1999 The mathematical modelling of cell kinetics in corneal epithelial wound healing. J. Theor. Biol. 197, 15-40.

Gardner, R. A. 1982 Existence and stability of travelling wave solutions of competition modelsa degree theoretic approach. J. Diff. Eqns 44, 343-364.

Gatenby, R. A. 1995 Models of tumor host interaction as competing populations-implications for tumor biology and treatment. J. Theor. Biol. 176, 447-455.

Gatenby, R. A. 1996 Application of competition theory to tumour growth: implications for tumour biology and treatment. Eur. J. Cancer A 32, 722-726.

Gatenby, R. A. \& Gawlinski, E. T. 1996 A reaction-diffusion model of cancer invasion. Cancer Res. 56, 5745-5753.

Höfer, T., Sherratt, J. A. \& Maini, P. K. 1995 Dictyostelium discoideum: cellular self-organization in an excitable biological medium. Proc. R. Soc. Lond. B 259, 249-257.

Hosono, Y. 1998 The minimal speed of travelling fronts for a diffusive Lotka-Volterra competition model. Bull. Math. Biol. 60, 435-448.

Huttenlocher, A., Lakonishok, M., Kinder, M., Wu, S., Truong, T., Knudsen, K. A. \& Horwitz, A. F. 1998 Integrin and cadherin synergy regulates contact inhibition of migration and motile activity. J. Cell. Biol. 141, 515-526.

Kan-on, Y. 1995 Parameter dependence of propagation speed of travelling waves for competitiondiffusion equations. SIAM J. Math. Analysis 26, 340-363.

Kan-on, Y. 1997 Fisher wavefronts for the Lotka-Volterra competition model with diffusion. Nonlin. Analysis TMA 28, 145-164.

Keller, E. F. \& Segel, L. A. 1971 Model for chemotaxis. J. Theor. Biol. 30, 225-234.

Kolmogoroff, A., Petrovsky, I. \& Piscounov, N. 1937 Etude de l'équation de la diffusion avec croissance de la quantité de matiére et sa application á un probléme biologique. Moscow Univ. Bull. Math. 1, 1-25.

Lotka, A. J. 1925 Elements of physical biology. Baltimore, MD: Williams \& Wilkins.

Marchant, B. P., Norbury, J. \& Perumpanani, A. J. 2000 Travelling shock waves arising in a model of malignant invasion. SIAM J. Appl. Math. 60, 463-476.

Michelson, S. \& Leith, J. 1991 Autocrine and paracrine growth factors in tumour growth: a mathematical model. Bull. Math. Biol. 53, 639-656.

Mimura, M. \& Fife, P. C. 1986 A 3-component system of competition and diffusion. Hiroshima Math. J. 16, 189-207.

Murray, J. D. 1989 Mathematical biology. Springer. 
Murray, J. D. \& Oster, G. F. 1984 Generation of biological pattern and form. IMA J. Math. Appl. Med. Biol. 1, 51-75.

Okubo, A. 1980 Diffusion and ecological problems. Springer.

Okubo, A., Maini, P. K., Williamson, M. H. \& Murray, J. D. 1989 On the spatial spread of the grey squirrel in Britain. Proc. R. Soc. Lond. B 238, 113-125.

Perumpanani, A. J., Sherratt, J. A. \& Norbury, J. 1997 Mathematical modelling of capsule formation and multinodularity in benign tumour growth. Nonlinearity 10, 1599-1614.

Pettet, G., Chaplain, M. A. J., McElwain, D. L. S. \& Byrne, H. M. 1996 On the role of angiogenesis in wound healing. Proc. R. Soc. Lond. B 263, 1487-1493.

Rothe, F. 1978 Convergence to travelling fronts in semilinear parabolic equations. Proc. R. Soc. Edinb. A 80, 213-234.

Sherratt, J. A. 1993 Cellular growth control and travelling waves of cancer. SIAM J. Appl. Math. 53, 1713-1730.

Sherratt, J. A. \& Murray, J. D. 1990 Models of epidermal wound healing. Proc. R. Soc. Lond. B 241, 29-36.

Sherratt, J. A. \& Nowak, M. A. 1992 Oncogenes, anti-oncogenes and the immune response to cancer. Proc. R. Soc. Lond. B 248, 261-271.

Skellam, J. C. 1951 Random dispersal in theoretical populations. Biometrika 38, 196-218.

Tang, M. M. \& Fife, P. C. 1980 Propagating fronts for competing species equations with diffusion. Arch. Ration. Mech. Analysis 73, 69-77.

van Vuuren, J. H. 1995 The existence of travelling plane waves in a general class of competition diffusion systems. IMA J. Appl. Math. 55, 135-148.

Volterra, V. 1926 Variazione fluttuazioni del numero d'individui in specie animali conviventi. Acad. Lincei. 2, 13-113. (English translation: Chapman, R. N. 1931 Animal ecology, pp. 409448. New York: McGraw-Hill.)

Ward, J. \& King, J. R. 1997 Mathematical modelling of avascular tumour growth. IMA J. Math. Appl. Med. Biol. 14, 39-69.

Wheldon, T. E. 1975 Mitotic autoregulation of normal and abnormal cells: alternative mechanisms for the derangement of growth control. J. Theor. Biol. 53, 421-433. 